\title{
New Onset Systemic Lupus Erythematosus Presenting with Massive Pericardial Effusion: A Case Report
}

\author{
Dorsa Kavandi', Majid Alikhani ${ }^{2}$, Sepideh Tahsini Tekantapeh ${ }^{3 *}$ \\ ${ }^{1}$ School of Medicine, Zanjan University of Medical Sciences, Zanjan, Iran. ${ }^{2}$ Rheumatology Research Center, Tehran University of \\ Medical Sciences, Tehran, Iran. ${ }^{3}$ Tabriz University of Medical Sciences, Tabriz, Iran.
}

\begin{abstract}
Systemic lupus erythematosus (SLE) is an autoimmune connective tissue disorder with multiple system involvements which commonly affects the cardiovascular system. Although pericarditis and pericardial effusion are prevalent cardiac manifestations in SLE, massive pericardial effusion as an initial presentation is unusual. We describe a 47-year-old woman who presented to the hospital with headache, dry cough, shortness of breath, and fatigue. According to the clinical, radiologic, echocardiographic and laboratory rheumatologic test findings, SLE was diagnosed and treatment with prednisolone, hydroxychloroquine, and mycophenolate mofetil was initiated. The patient improved clinically, and follow-up echocardiography showed a reduction in the effusion volume compared with previous tests within the preceding 6 months. In patients with cardiopulmonary symptoms, especially when other organ involvement is seen, screening for autoimmune systemic diseases such as SLE should be considered. To achieve rapid recovery and prevent life-threatening complications, early diagnosis and treatment are essential.
\end{abstract}

Keywords: SLE, massive pericardial effusion, echocardiography

\section{Introduction}

Systemic lupus erythematosus (SLE) is an autoimmune connective tissue disorder with multiple system involvement which commonly affects the cardiovascular system [1]. Any part of the cardiovascular system can be affected including myocardium, pericardium, coronary arteries, endocardium, and conduction system $[1,2]$. Cardiac involvement, especially pericarditis and pericardial effusion, are prevalent cardiac manifestations in SLE; however, massive pericardial effusion as an initial presentation is unusual $[1,2]$. In this report we describe a 47-year-old Iranian woman who presented to the emergency department with massive pericardial effusion. The diagnosis of SLE was confirmed during her hospitalization. The patient improved clinically with evidence of decline in the volume of pericardial effusion after treatment with prednisolone, hydroxychloroquine, and mycophenolate mofetil over a 6-month period.

\section{Case Presentation}

A 47-year-old Iranian woman with a one-year history of hypothyroidism came to the hospital with headache, dry cough, shortness of breath, and fatigue lasting for 2 weeks but no complaints of photosensitivity, skin rash, arthralgia,
Raynaud's phenomenon, palpitation, or dizziness. Upon physical examination, the patient was conscious and oriented with a good general condition and no respiratory distress. She had a hypertensive crisis (blood pressure $=170 / 110$ ), tachycardia (pulse rate $=$ 110 ), tachypnea (respiratory rate $=25$ ), low-grade fever (body temperature $=37.8$ oral). Cardiac sounds were muffled and her lungs were clear in auscultation. No paradoxical pulse was detected, and the jugular vein was not prominent.Examination of skin and mucosal membranes, eyes, joints, and nervous system was unremarkable. Laboratory tests revealed pancytopenia (platelet count: 111,000; hemoglobin = 10.6; white blood cells (WBCs): 2600) and elevated erythrocyte sedimentation rate $(\mathrm{ESR}=78 \mathrm{~mm} /$ first hour),hematuria and proteinuria (urinalysis (U/A): blood2+; red blood cell $=20-25$; protein $=1+$ ). Normal thyroid function tests (thyroid stimulating hormone (TSH), free $\mathrm{T}_{4}$ and $\mathrm{T}_{3}$ )with positive titer of anti-thyroid peroxidase antibody (Anti-TPO antibody) were reported. In the electrocardiogram,sinus tachycardia and a low voltage pattern were detected (Figure 1). Cardiomegaly was found on chest X-ray (Figure 2).

Personal non-commercial use only. Rheumatology Research Journal. Copyright $($ 2020. All rights reserved.

*Corresponding Author: Sepideh Tahsini Tekantapeh Tabriz University of Medical Sciences, Tabriz, Iran. E-mail: tahsinis@tbzmed.ac.ir, Telefax: +98-914-382-6012.

Received: 25 December 2019; Accepted: 03 February 2020 




Figure 1. ECG of patient showing sinus tachycardia and low voltage qrs complexes. 

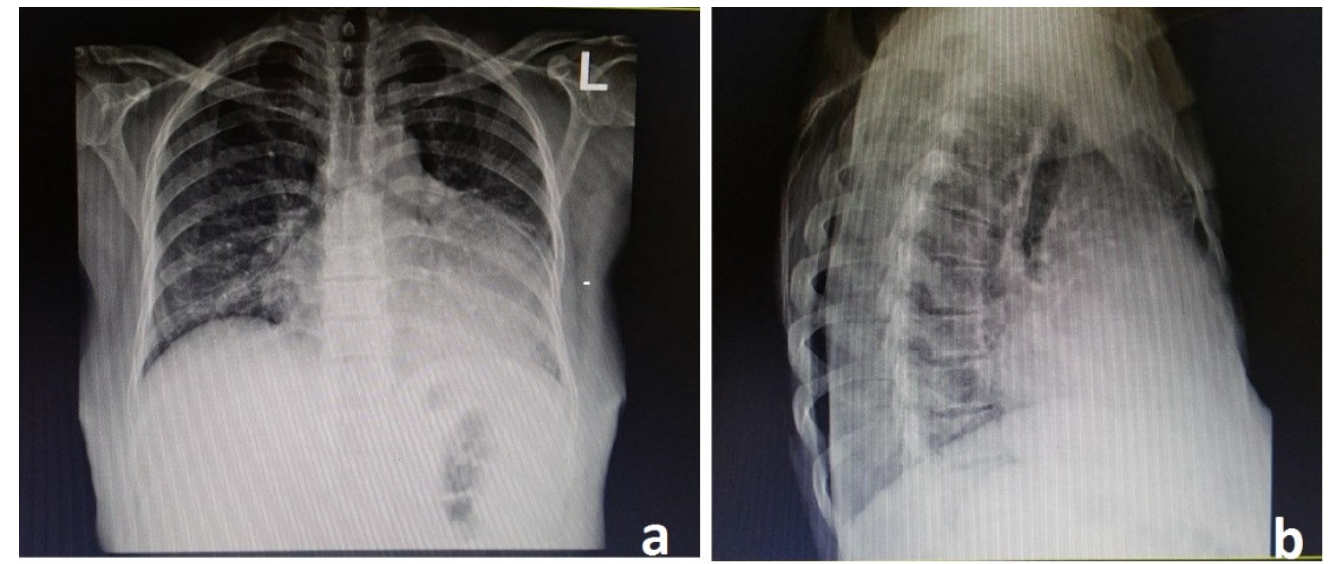

Figure 2. Chest X-ray of patient with dry cough and dyspnea showing cardiomegaly in favor of pericardial effusion.

Because of dyspnea and cardiomegaly echocardiography was done which showed massive pericardial effusion without vegetation. Spiral lung and mediastinal computed tomography scan revealed massive pericardial effusion without pleural effusion (Figure 3).

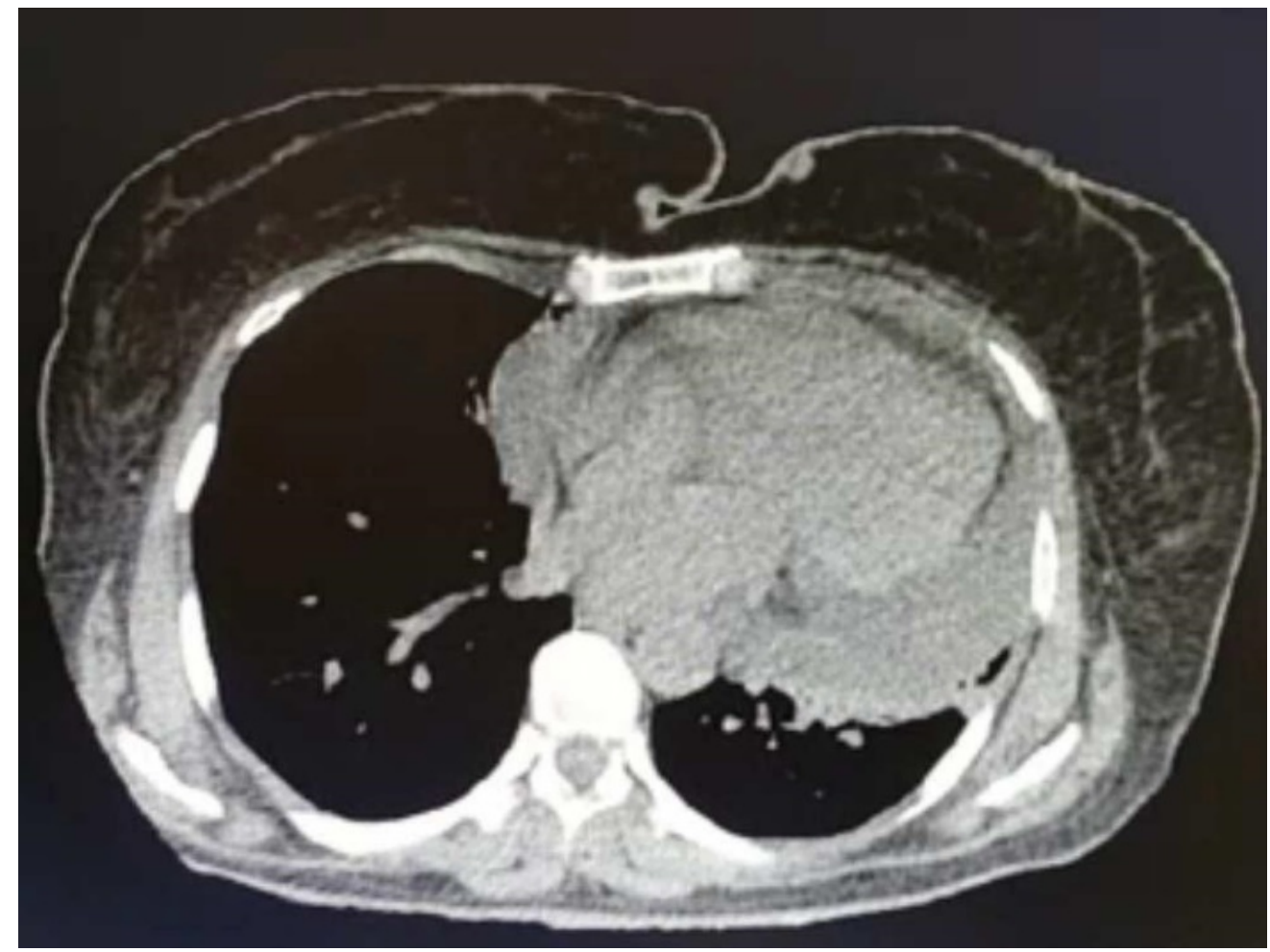

Figure 3. Chest computed tomography scan (CT) showing pericardial effusion.

The patient was transferred to the intensive care unit (ICU) for close monitoring. Because of her pancytopenia, a peripheral blood smear (PBS) was requested, and the results indicated enlarged platelets and anisocytosis with an approximate platelet count $=140,000 /$ microliter, erythrocyte hypochromia and anisopoikilocytosis. It was polymorphonuclear leukocytes (PMN) dominant, and no atypical cell was seen. Bone marrow aspiration and biopsy revealed hyper-cellular marrow with some megaloblastic changes but no malignant cells. Abdominopelvic ultrasound showed that the spleen was enlarged ( $\operatorname{span}=$
$139 \mathrm{~mm}$ ); other organs were normal. Because of proteinuria in U/A, 24-hour urine protein was measured and was 110 milligrams (mg) (below 500), so the kidney biopsy was canceled. After that, according to investigations, suspicious infectious diseases such as tuberculosis, brucellosis, hepatitis B, hepatitis C, and AIDS were ruled out. Tuberculosis was ruled out based on the negative PPD (purified protein derivative) and IGRA (interferon gamma release assay) tests. Based on the patient's pancytopenia, proteinuria, hematuria, increased ESR, and serositis as well as the ruling out of neoplastic 
and probable infectious diseases, connective tissue disorders (particularly SLE) were highly probable. The patient was then started on 60 milligrams prednisolone daily, and rheumatologic tests were requested. Serum complement components $(\mathrm{C} 3, \mathrm{C} 4$, and $\mathrm{CH} 50)$ were in the normal range. The results of rheumatologic tests are shown in Table 1.

Table 1. Rheumatologic laboratory findings of the patient in favor of SLE

\begin{tabular}{cll}
\hline Test & Result & Normal Range \\
\hline Fluorescent antinuclear antibody(ANA) (speckled pattern) & $1 / 160$ & $<1 / 40$ \\
Anti-double stranded DNA antibody & $>200$ & $<75.0 \mathrm{IU} / \mathrm{mL}$ \\
Anti-Ro antibodies & $>200$ & $<1 \mathrm{U}$ \\
\hline
\end{tabular}

SLE was proven based on 4 of 17 SLE 2012 SLICC (Systemic Lupus International Collaborating Clinics) diagnostic criteria, including pericardial effusion, leukopenia, positive ANA, and elevated anti-dsDNA. Treatment with prednisolone was continued, and $400 \mathrm{mg}$ hydroxychloroquine and 3 grams mycophenolate mofetil were added to the patient's therapeutic regimen. The patient improved clinically, and her echocardiography during the 6-month follow-up period showed a significant reduction in effusion volume compared with previous tests. The patient was discharged from the hospital with a prescription for $60 \mathrm{mg}$ prednisolone daily with tapering recommendation, 3 gr mycophenolate mofetil, and hydroxychloroquine 400mg daily.

\section{Discussion}

SLE is an autoimmune systemic connective tissue disorder. The production of autoantibodies and deposition of immune complexes within several tissues lead to various disease manifestations. Cardiovascular system involvement has been reported in up to $50 \%$ of SLE patients $[2,3]$. Any part of the heart can be involved in the disease course. The pericardium and endocardium are commonly affected areas with reported prevalence rates of $53-74 \%$ and $48-50 \%$, respectively [3]. Among the cardiovascular symptoms, pericarditis and pericardial effusion are the most prevalent manifestations. The incidence of SLE-related pericarditis and pericardial effusion has been reported as $75 \%$ and $40 \%$, respectively $[1,2]$. Cardiac involvement is rarely detected as an initial presenting feature in SLE, and pericardial effusion is usually mild, small, and insignificant in SLE patients; massive symptomatic pericardial effusion and cardiac tamponade, however, are also possible as initial presentations in this disease [2]. Cardiac complications are important leading causes of morbidity and mortality in patients with SLE. In this case, a middle-aged woman with no underlying cardiopulmonary diseases presented with fatigue, headache, and dry cough which had progressed to shortness of breath in two weeks. According to her medical history, physical examination, and initial laboratory investigations which showed pancytopenia and pericardial effusion, the possibility of systemic diseases was highly probable. On the other hand, pericardial effusion can be a complication of poorly controlled hypothyroidism. The amount of pericardial effusion is associated with disease severity, but pericardial effusion in this setting is rarely massive [4]. Thus, the pericardial effusion in the current case may be related to hypothyroidism which was proven in her past medical history. Thyroid function tests (TSH, free $\mathrm{T}_{4}$ and $\mathrm{T}_{3}$ ) were done, and all results were in normal range. Massive pericardial effusion in addition to pancytopenia suggest the possibility of systemic rheumatologic diseases, specifically SLE (at the top of diagnosis). Further investigations for SLE (positive ANA and anti-ds-DNA levels) supported this diagnosis, and SLE 2012 SLICC diagnostic criteria was fulfilled with 4 of 17 (pericardial effusion, leukopenia, positive ANA and positive anti-dsDNA) (2). After confirming the diagnosis of SLE, the association between SLE and hypothyroidism as well as the roles of autoimmunity and thyroid autoantibodies were discussed. The current patient had not been previously screened for autoimmune hypothyroidism. Her anti-TPO Ab was positive, but it was not possible to measure anti-thyroglobulin (anti-Tg) or thyrotropin receptor (TRAb) in our hospital. According to previous research, a higher prevalence of thyroid disorders, especially hypothyroidism, were detected in SLE patients. Thyroid disorders were reported in $25.3 \%$ (vs. $9.4 \%$ in the control group) of SLE patients [5]. The majority of studies have shown the association between SLE and hypothyroidism. The incidence of hypothyroidism in SLE patients is reported to be $21.5 \%$ [5]. According to recent studies, a high prevalence of autoimmune thyroid diseases, reportedly $11.4 \%$, has been found in SLE patients. The association between autoimmune thyroid diseases and SLE has been studied since 1961. Hijmans et al. and White et al. reported the first cases of Hashimoto's thyroiditis (HT) in SLE patients [6]. Several studies have shown various results about the presence of anti-thyroid antibodies in SLE patients. Despite the most common results, Domingues et al. demonstrated no association between anti-thyroid antibodies and SLE [5]. Increased erythrocyte sedimentation rate (ESR) levels, which are closely related to disease activity, have been detected in most SLE cases. It also has a greater correlation with positivity of antidsDNA antibodies [7]. ESR is an inflammatory marker which is used in the follow-up period. As estimated in the current case, ESR was increased. Therefore, after treatment, it decreased in amounts. Also as estimated, Creactive protein (CRP) levels were not increased in the current case. CRP is another inflammatory marker which 
increases slightly in active SLE. Significant elevation of CRP was noted by some studies in patients with serosal inflammation and musculoskeletal involvement [8]. Glucocorticoid therapy has been proven to be an effective treatment of pericardial effusion in SLE patients. Other therapeutic options include immunosuppressants, intrapericardial injection of glucocorticoid, and ultrasoundguided pericardiocentesis [9]. The current case responded to treatment with 60 milligrams prednisolone daily and showed a reduction in pericardial effusion, unlike a case reported by Kamata et al. Navreet Sharda also reported the successful treatment of an 11-year-old girl with massive hemorrhagic pericardial effusion with steroids, which led to an improvement in symptoms along with a rising trend in platelet count [10]. Because of the possibility of developing from pericardial effusion to cardiac tamponade, early treatment is important. In conclusion, systemic lupus erythematosus should be kept in mind in patients with cardiopulmonary symptoms, especially when other organ involvement is seen, even without previous diagnosis or a history suggestive of systemic lupus erythematosus [10]. To achieve rapid recovery and prevent life-threatening complications such as pericardial effusion leading to tamponade, early diagnosis and treatment are essential.

\section{Conclusion}

Systemic lupus erythematosus should be considered in patients with cardiopulmonary symptoms as a first presentation, especially when other organ involvement is seen, even without previous diagnosis or a history suggestive of SLE. To achieve rapid recovery and prevent life-threatening complications such as pericardial effusion leading to tamponade, early diagnosis and treatment are essential.

\section{Acknowledgments}

This report has not been presented elsewhere. We wish to thank all of the co-medical staff involved in the treatment.

\section{Conflict of interest}

The authors declare they have no conflicts of interest in association with the present study.

\section{References}

1. Bezwada P, Quadri A, Shaikh A, Ayala-Rodriguez C, Green S. Myopericarditis and Pericardial Effusion as the Initial Presentation of Systemic Lupus Erythematosus. Case Rep Med 2017; 2017:6912020. doi: $10.1155 / 2017 / 6912020$.

2. Cheo SW, Low QJ. Massive pericardial effusion - An uncommon initial presentation of systemic lupus erythematosus (SLE). Med J Malaysia 2019; 74(5):43940.

3. Tian DG, Jacobs AK, Benjamin EJ. A 19-year-old female with tamponade and systemic lupus erythematosus (SLE). Int J Cardiol 2015; 179:1-2. doi: 10.1016/j.ijcard.2014.10.054.

4. Purkait R, Prasad A, Bhadra R, Basu A. Massive pericardial effusion as the only manifestation of primary hypothyroidism. J Cardiovasc Dis Res 2013; 4(4):24850. doi: 10.1016/j.jcdr.2014.01.001.

5. Domingues SL, Gonçalves FT, Jorge M, Limongi JE, Ranza R, Jorge PT. High prevalence of hypothyroidism in systemic lupus erythematosus patients without an increase in circulating anti-thyroid antibodies. Endocr Pract 2017; 23(11):1304-10. doi: 10.4158/ep161664.Or.
6. Kumar K, Kole AK, Karmakar PS, Ghosh A. The spectrum of thyroid disorders in systemic lupus erythematosus. Rheumatol Int 2012; 32(1):73-8. doi: 10.1007/s00296-010-1556-5.

7. Stojan G, Fang H, Magder L, Petri M. Erythrocyte sedimentation rate is a predictor of renal and overall SLE disease activity. Lupus 2013; 22(8):827-34. doi: 10.1177/0961203313492578.

8. Aringer M. Inflammatory markers in systemic lupus erythematosus. J Autoimmun 2020; 110:102374. doi: 10.1016/j.jaut.2019.102374.

9. Kamata Y, Iwamoto M, Aoki Y, Kishaba Y, Nagashima T, Nara H. et al. Massive intractable pericardial effusion in a patient with systemic lupus erythematosus treated successfully with pericardial fenestration alone. Lupus 2008; 17(11):1033-35. doi: $10.1177 / 0961203308089437$.

10. Sharda N. Cardiac tamponade as an initial manifestation of systemic lupus erythematosus in a child. Cardiol Young 2014; 24(1):172-74. doi: $10.1017 / \mathrm{s} 1047951112001916$. 
